\title{
Evaluación de la velocidad, agilidad y fuerza en jóvenes jugadores de pádel Speed, agility, and strength assessment in young padel players
}

\author{
Bernardino Javier Sánchez-Alcaraz Martínez, Vicente Orozco Ballesta, Javier Courel-Ibáñez, Alejandro Sánchez Pay
} Universidad de Murcia (España)

\begin{abstract}
Resumen. El objetivo de este estudio es evaluar la velocidad de desplazamiento, la fuerza, y la agilidad en jóvenes jugadores de pádel, y analizar las diferencias en función del género. Los participantes de la investigación fueron 17 jugadores de pádel de nivel avanzado (8 chicos y 9 chicas) con edades comprendidas entre los 11 y los 16 años. Los jugadores completaron el test del hexágono, el de velocidad lineal de 10 y 20 metros y el de lanzamiento de balón medicinal. Los resultados mostraron que los jugadores de pádel masculinos presentaron unos tiempos significativamente inferiores que las participantes de género femenino en velocidad de sprint lineal, fuerza de tren superior y velocidad de cambio de dirección. Las variables de velocidad y velocidad de cambio de dirección correlacionan significativa y positivamente sí, mientras que la variable de fuerza correlaciona significativa y negativamente con las variables de velocidad de desplazamiento y velocidad de cambio de dirección.
\end{abstract}

Palabras clave: Pádel, Fuerza, Velocidad, Agilidad.

Abstract. The aim of this study is to evaluate lineal speed, strength, and agility in young padel players, and to analyse the differences by gender. The research participants were 17 advanced level padel players (8 boys and 9 girls) aged 11 to 16 years. Players completed the hexagon test, the 10-meter and 20-meter linear speed tests, and the medical ball throwing test. Results show that male paddle players present better scores than female participants in linear velocity, upper train force, and speed of change of direction. The variables of speed and change direction speed correlate significantly and positively, while strength correlates significantly and negatively with speed and change direction speed.

Keywords: Padel, Strength, Speed, Agility.

\section{Introducción}

El pádel es un deporte de reciente creación que se practica en cerca de 30 países (Federación Internacional de Pádel, 2016; Sánchez-Alcaraz, 2013). En España, es uno de los 10 deportes más practicados en sólo dos décadas de historia, (García-Ferrando, y Llopis, 2010; Ministerio de Educación, Cultura y Deportes, 2015) y con un crecimiento cada vez mayor en número de licencias y deinstalaciones (Courel-Ibáñez, SánchezAlcaraz, García-Benítez, y Echegaray, 2017; Muñoz et al., 2016). Esta rápida evolución ha ido acompañada de un aumento en las publicaciones científicas específicas de este deporte, con especial interés en la descripción de las demandas y los requerimientos energéticos de la competición (Sánchez-Alcaraz, Cañas y Courel-Ibáñez, 2015; SánchezAlcaraz, Courel-Ibáñez, y Cañas, 2018). Sin embargo, existen carencias en el conocimiento de las capacidades físicas de jugadores de pádel.

La tipología de los aspectos técnico-tácticos y temporales del pádel, a excepción del servicio, se caracteriza por estímulos y acciones de rápida ejecución con el objetivo de golpear la pelota al otro lado de la pista de manera precisa (Sánchez-Alcaraz, y Gómez-Mármol, 2015). Este conjunto de acciones se repiten a lo largo de partidos que se prologan más allá de la hora, por lo que los jugadores necesitan unos niveles de condición física que les permita ser eficaces de acuerdo a las demandas de la competición (Courel-Ibáñez, Sánchez-Alcaraz, y Cañas, 2015, 2017; Muñoz et al., 2017; Torres-Luque, Ramírez, CabelloManrique, Nikolaidis, y Alvero-Cruz, 2015). Diferentes estudios han mostrado que las acciones de juego y los aspectos temporales del pádel pueden variar en función de la edad, el género de los deportistas o el tipo de entrenamiento, por lo que las exigencias físicas pueden ser diferentes según el perfil del practicante(Sánchez-Alcaraz, 2014a; 2014b; SánchezAlcaraz, Sánchez-Pay, Gómez-Mármol, Molina-Saorín y BazacoBelmonte, 2017). Este dato resulta de vital importancia a la hora de diseñar y planificar entrenamientos y estrategias de competición de manera precisa e individualizada (Glazier, 2010).

A lo largo de un partido de pádel un jugador realizar una gran cantidad de golpeos de manera unilateral (García Benítez, Courel-Ibáñez, Pérez-Bilbao, y Felipe, In press). Esta resistencia crónica a la fatiga provoca que el mayor índice de lesiones se produzca en el tren superior, especialmente en codos y hombros (Castillo-Lozano, 2017). Estudios

Fecha recepción: 12-10-17. Fecha de aceptación: 01-02-18 Bernardino Javier Sánchez-Alcaraz Martínez bjavier.sanchez@um.es previos en tenis sugieren que un entrenamiento de la fuerza del tren superior es indispensable tanto para mantener un adecuado ritmo de competición como para evitar dolencias y abandonos de la práctica (Maquirriain, Baglione, y Cardey, 2016).

La elevada frecuencia de los golpeos y las reducidas dimensiones de la pista en pádel exigen al jugador realizar desplazamientos de corta distancia y rápida intensidad que le permitan llegar a tiempo a golpear la pelota en las mejores condiciones posibles (Courel-Ibáñez, SánchezAlcaraz y Cañas, 2016; Courel-Ibáñez, Sánchez-Alcaraz, y Cañas, 2017). Además, una de las características específicas del pádel es la existencia de paredes sobre las que la pelota puede rebotar, lo que genera la aparición de giros y pivotes previos al golpeo de la bola, especialmente en las esquinas del fondo de la pista (Courel-Ibáñez, Sánchez-Alcaraz, y Muñoz, 2017; Priego et al., 2013). Por tanto, una adecuada velocidad de desplazamiento y una mejor habilidad para cambiar de dirección parecen ser importantes en pádel.

En resumen, existe un creciente interés en el estudio de las demandas de la competición y los requerimientos energéticos en pádel (SánchezAlcaraz et al., 2015; 2018). Sin embargo, son notables las carencias de investigaciones que muestren resultados sobre valoraciones de la condición física jugadores de pádel. El uso de test como el sprint de $10 \mathrm{~m} / 20 \mathrm{~m}$ y el test del hexágono han sido ampliamente utilizados en deportes similares como el tenis (Fernández-Fernández, Ulbricht, y Ferrauti, 2014; Roetert, y Ellenbecker, 2007). Asimismo, el lanzamiento de balón medicinal se muestra como un método eficaz y muy utilizado para valorar la fuerza del tren superior en deportes de raqueta(Earpy Kreaemer, 2010; Fernández-Fernández et al, 2014). Una correcta planificación del entrenamiento del jugador debe de constar de pruebas (valoraciones a través de test) y seguimiento (Roetert y Ellenbecker, 2008). Por lo tanto, el objetivo de este estudio será evaluar la velocidad de desplazamiento, la fuerza, y la agilidad en jóvenes jugadores de pádel, y analizar las diferencias en función del género.

\section{Método}

\section{Participantes}

Los participantes de la investigación fueron 17 jugadores de pádel con edades comprendidas entre los 11 y los 16 años, de los cuales 8 eran chicos (14.12 \pm 1.24 años) y 9 chicas (14.33 \pm 1.24 años). Todos los jugadores realizaban una práctica regular de pádel de más de dos días en semana durante al menos dos años y participaban en un mínimo de 10 torneos durante la temporada. 


\section{Medidas e instrumentos}

Se registraron cuatro medidas por participante a través de test de campo (Figura 1) ampliamente utilizados en la evaluación de jóvenes y en deportes de raqueta (Fernández-Fernández et al, 2014) debido a su fiabilidad y sencillez.

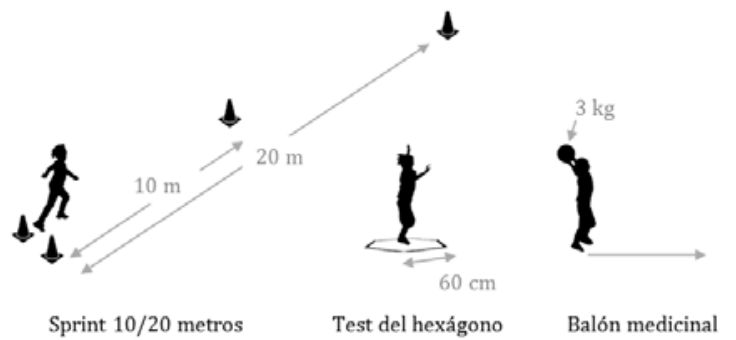

Figura 1. Representación de los tres test de campo empleados para valorar la agilidad, velocidad y fuerza en jóvenes jugadores de pádel.

Velocidad de desplazamiento: Se utilizaron los test de sprint de 10 y 20 metros, con unos valores de fiabilidad de .97 en tenistas (SánchezPay, Torres, y Palao, 2011). El participante se sitúa en posición de salida alta tras la línea de salida. Ala señal del controlador, deberá recorrer la distancia de 10 o 20 metros en el menor tiempo posible, hasta sobrepasar con el cuerpo la línea de llegada. Se contabilizó el mejor tiempo en segundos y centésimas de segundo de dos intentos.

Velocidad de cambio de dirección. Se utilizó el test del hexágono (Reid, Quinn, y Crespo, 2003). El participante se sitúa el centro de un hexágono de 60 centímetros de lado. Ala señal del controlador, deberá saltar con ambos pies hacia delante por encima de la línea y volver al centro del hexágono a la máxima velocidad posible. Mirando en la misma dirección, irá repitiendo la acción en cada lado del hexágono en sentido de las agujas del reloj. La prueba terminó cuando el participante completó tres vueltas completas y sitúe los pies en el centro del hexágono. Se contabilizó el mejor tiempo en segundos y centésimas de segundo de dos intentos.

Fuerza del tren superior: Se evaluó la fuerza explosiva a través de un test de lanzamiento frontal de balón medicinal de $3 \mathrm{~kg}$ de peso a la mayor distancia horizontal posible. Los participantes siguieron las siguientes instrucciones (Rivilla-García, Navarro, Grande, y Sampedro, 2012) pies colocados a la anchura de los hombros, cuerpo orientado en la dirección del lanzamiento y balón adaptado con las dos manos simétricamente debajo de la cadera. El lanzamiento consiste en elevar el balón medicinal con ambas manos por encima y detrás de la cabeza, extender el tronco flexionando los codos y rodillas y, finalmente, realizar una movimiento explosivo hacia delante (flexionando el tronco y extendiendo los hombros, codos y rodillas) para lanzar el balón a la mayor distancia horizontal posible. Se permite elevar los talones pero no despegar los pies del suelo. El lanzador no puede traspasar la línea de lanzamiento. Se realizaron pruebas de familiarización hasta que los participantes consiguieron una cadena cinética eficaz, simulando el gesto técnico del remate en pádel. Se registraron la distancia más lejana en centímetros tras dos lanzamientos.

\section{Procedimiento}

Todos los deportistas fueron informados sobre el procedimiento del estudio, y entregaron un consentimiento informado para participar en el mismo. Se citó a todos los jugadores que iban a participar en las pruebas a la misma hora del día, con una temperatura de $25^{\circ} \mathrm{C}$. En primer lugar se realizó un periodo de 10 minutos de calentamiento dirigido por un licenciado en Ciencias de la Actividad Física y del Deporte. Inmediatamente al término del calentamiento, los jugadores completaron los test de condición física en el siguiente orden: sprint de 10 metros, sprint de 20 metros, hexágono y lanzamiento de balón medicinal. Los datos se recogieron a través de una de una hoja de registro durante el desarrollo de las pruebas. Cada serie se filmó con una cámara de vídeo de alta resolución (GoPro HD Hero 3+, GoPro Inc., San Mateo, California). Poste- riormente, dos observadores experimentados extrajeron el tiempo y distancia de cada test.

\section{Análisis de datos}

El análisis descriptivo de los datos incluyó el cálculo de media y desviación típica ( $\mathrm{M} \pm \mathrm{DT}$ ), y mínimo y máximo de las variables estudiadas. El pequeño tamaño de la muestra recomendó el uso del test de Shapiro-Wilk para contrastar la normalidad de los datos obtenidos para cada variable. Se aplicó la prueba U de Mann-Whitney para la comparación de medias entre grupos según el género de los jugadores, considerando un nivel de significación de $<<0.05$. Se calculó el cálculo del tamaño del efecto de las comparaciones de medias través de eta al cuadrado ( $\eta 2$ ), con la fórmula ๆ2=Z2/N-1 (Tomczak y Tomczak, 2014). Finalmente, se estudiaron las correlaciones entre las diferentes variables de condición física a través del cálculo del coeficiente r de Pearson. Los análisis fueron realizados con el software SPSS para Windows (Versión 20.0. Armonk, NY:IBM Corp.).

\section{Resultados}

La tabla 1 muestra los estadísticos descriptivos (media, desviación típica, mínimo y máximo) de cada uno de los test de condición física para el total de los jugadores de pádel.

La tabla 2 muestra la comparación de las puntuaciones en los test en función del género de los deportistas. Los chicos mostraron mejores puntuaciones en cada uno de los test, encontrando diferencias significativas en la fuerza del tren superior $(\mathrm{p}<0.01)$ en la velocidad de sprint de 10 y 20 metros $(\mathrm{p}<0.05)$. Por el contrario, los valores de velocidad de cambio de dirección fueron muy similares.

En la tabla 3 se expone la relación entre las diferentes medidas. Todas las variables mostraron una correlación significativa entre sí. Las variables de sprint 10 y 20 metros y cambio de dirección correlacionaron positivamente (a mayor velocidad de sprint, mayor velocidad de cambio de dirección). Por el contrario, la fuerza del tren superior mostró una correlación negativa con las variables de velocidad de sprint y velocidad de cambio de dirección (i.e., los participantes con mayores índices de fuerza en el tren superior fueron más lentos y menos ágiles).

\section{Discusión}

El objetivo de este estudio fue evaluar la velocidad de desplazamiento, la agilidad y la fuerza en jóvenes jugadores de pádel y analizar las diferencias en función del género. Anivel general, los jugadores de pádel jóvenes, en las pruebas de velocidad de sprint lineal de 10 y 20 metros mostraron tiempos entre 0.5 y 0.7 segundos más lentos en comparación con los jugadores de fútbol de su misma edad (Buchheit,

Tabla 1.

Puntuaciones en los test de velocidad, de cambio de dirección y de fuerza para el total de los jugadores de pádel.

Variable

Sprint 10 metros (s)

Sprint 20 metros (s)

Canbio de dirección (s)

tren superior $(\mathrm{cm})$

Valores expresados en media y desviación típica (M \pm DT)

$\begin{array}{cc}M \pm D T & \text { Min - Max } \\ 2.71 \pm 0.25 & 2.24-3.23 \\ 4.09 \pm 0.39 & 3.50-4.94 \\ 4.87 \pm 2.71 & 11.85-21.45\end{array}$

Tabla 2 .

Puntuaciones en los test de velocidad, de cambio de dirección y de fuerza en función del género de los jugadores de pádel.

\begin{tabular}{|c|c|c|c|c|}
\hline \multirow[t]{2}{*}{ Variable } & $\begin{array}{l}\text { Masculino } \\
(\mathrm{n}=8)\end{array}$ & $\begin{array}{l}\text { Femenino } \\
(\mathrm{n}=9)\end{array}$ & \multirow[t]{2}{*}{$p$} & \multirow[t]{2}{*}{$T D E$} \\
\hline & $M \pm D T$ & & & \\
\hline Sprint 10 metros (s) & $2.56 \pm 0.26$ & $2.84 \pm 0.17$ & .008 & .42 \\
\hline Sprint 20 metros (s) & $3.87 \pm 0.30$ & $4.29 \pm 0.37$ & .021 & .33 \\
\hline Cambio de dirección (s) & $14.39 \pm 2.32$ & $15.39 \pm 3.06$ & .606 & 02 \\
\hline Fuerza del tren superior (cm) & $625 \pm 9.70$ & $423 \pm 6.70$ & $<.001$ & .71 \\
\hline
\end{tabular}

$\begin{array}{lrrr}\text { Fuerza del tren superior }(\mathrm{cm}) & 625 \pm 9.70 & 423 \pm 6.70 & <.001\end{array}$

Tabla 3.

\begin{tabular}{|c|c|c|c|c|c|}
\hline & & $10 \mathrm{~m}$ & $20 \mathrm{~m}$ & CD & FTS \\
\hline \multirow{2}{*}{ Sprint 10 metros $(10 \mathrm{~m})$} & Correlación & & .836 & .585 & -.836 \\
\hline & Significación & & $<.001 * *$ & $.014^{*}$ & $<.001^{* *}$ \\
\hline Sprint 20 metros (20m) & $\begin{array}{l}\text { Correlación } \\
\text { Significación }\end{array}$ & & & .667 & -.766 \\
\hline Cambio de dirección (CD) & $\begin{array}{l}\text { Correlación } \\
\text { Significación }\end{array}$ & & & & $\begin{array}{l}-.567 \\
.018^{*}\end{array}$ \\
\hline Fuerza de tren superior (FTS) & $\begin{array}{l}\text { Correlación } \\
\text { Significación }\end{array}$ & & & & \\
\hline
\end{tabular}


Méndez-Villanueva, Delhomel, Brughelli, y Ahmaidi, 2010; FrancoMárquez et al., 2015; Junior, Palma, Imbiriba, Assis, y Barbosa, 2015). En comparación con sus homólogos tenistas, los jugadores de pádel de este estudio mostraron tiempos entre 0.4-0.5 segundos más lentos en los chicos, y entre 0.7-0.8 segundos en las chicas (Fernández-Fernández et al., 2014). Con respecto a la velocidad de cambio de dirección, los datos obtenidos por los jugadores de pádel mostraron tiempos ligeramente superiores (0.1-0.2 segundos) en comparación con tenistas de alto rendimiento de edades similares (Roetert y Ellenbecker, 2007). En este sentido, parece que las demandas físicas de la competición en pádel en etapas de formación no requieren que los jugadores sean tan rápidos o ágiles como en otros deportes, debido posiblemente a que las dimensiones de la pista son más reducidas y la alta frecuencia de golpeos no permite realizar tantos desplazamientos (Priego et al., 2013). Sin embargo, si parece importante que sean capaces de desarrollar cualidades tácticas como una buena colocación en la pista o una correcta lectura de las trayectorias de la pelota (Courel-Ibáñez, Sánchez-Alcaraz, y Muñoz, 2017).

Las comparaciones por género revelaron tiempos significativamente inferiores en chicos que en chicas en sprint y cambio de dirección. Estos datos son contrarios a los de algunas investigaciones recientes en escolares de entre 6-8 años (Yanci y Los Arcos, 2015; Yanci, Los Arcos, Grande, y Cámara, 2014) o en jugadores de tenis (Sánchez-Alcaraz, López-Sánchez, y Pagán, 2016), aunque estudios anteriores afirmaron que las diferencias de género en el desarrollo motor aparecen durante la totalidad del proceso madurativo de los niños (Raudsepp y Paasuke, 1995). Parece por tanto que en edades tempranas ( $<8$ años) las diferencias en la velocidad y cambio de dirección entre sexos son mínimas, hasta una determinada edad ( $>12$ años) donde estas diferencias se manifiestan de forma significativa (Amusa, Goon, y Amey, 2010). No obstante, existen diferencias en el estilo de juego en pádel entre el género masculino y femenino, ya que aunque la duración de los puntos es similar, los chicos realizan un número significativamente mayor de golpeos quelas chicas (Courel-Ibáñez y Sánchez-Alcaraz, 2017; SánchezAlcaraz, 2014b), principalmente porque estas últimas utilizan un número mayor de globos (Muñoz-Marín et al., 2017; Torres-Luque et al., 2015). Como consecuencia, la velocidad de juego en el pádel masculino es mayor, por lo que se requiere una mayor velocidad y agilidad para hacer frente a las demandas de la competición.

Los resultados obtenidos de las correlaciones sugieren que la velocidad de sprint lineal está altamente relacionada con la velocidad de cambio de dirección en jóvenes jugadores de pádel. Esta relación ha sido muy explorada en otros deportes y se han obtenido resultados amplios y contradictorios (Buttifant, Graham, y Cross, 2001; Little y Williams, 2005; Vescovi y McGuigan, 2008; Young, Hawken, y McDonald, 1996; Young, McDownell, y Scarlett, 2001). Young et al., (2001) observaron correlaciones bajas y no significativas de 0,27 y 0,19 entre sprint de $20 \mathrm{~m}$ y pruebas de cambio de dirección $3 \times 90^{\circ}$ y $3 \times 120^{\circ}$, respectivamente en deportistas universitarios. Del mismo modo, Buttifant et al. (2002) observaron un bajo coeficiente de determinación (5-10\%) entre la prueba de sprint de 20 m y pruebas de cambio de dirección con zigzag en jugadores de fútbol. Además, Little y Williams (2005) encontraron una relación baja entre un sprint con salida lanzada de $20 \mathrm{~m}$. y una prueba de cambio de dirección con zig-zag en jugadores de fútbol profesionales. Sin embargo, resultados más recientes de Vescovi y McGuigan (2008) revelaron algunas correlaciones moderadamente fuertes entre varias pruebas de sprint con salida detenida y sprints con salida lanzada y el test de agilidad de Illinois en atletas femeninas. Estos resultados tan variados pueden deberse a las diferencias metodológicas en los diferentes estudios, destacando principalmente que los test de sprint lineal y velocidad de cambio de dirección utilizados han sido diferentes y que el tipo de participantes también ha variado en edad y tipo de deporte practicado. Por otro lado, el test de fuerza correlaciona negativa y significativamente con los test de velocidad lineal y de cambio de dirección, por lo que parece que los jugadores más fuertes son menos ágiles y rápidos. Estos datos no coinciden con el estudio de Hernández (2003), quien estudió la relación existente entre la velocidad, fuerza y potencia, y no encontró correlación significativa entre la variable de fuerza y velocidad.

Este estudio presenta una serie de limitaciones que deben ser tenidas en cuenta a la hora de interpretar los resultados. En primer lugar, la escasa muestra ha impedido conocer las diferencias en los niveles de condición física en función de la edad de los jugadores, por lo que futuros estudios podrían ampliar el tamaño muestral y analizar la influencia de otro tipo de variables como el nivel de juego. Además, podría resultar interesante incluir otro tipo de test de campo para medir la velocidad de cambio de dirección, como el T-test, o realizar medidas específicas como la velocidad de lanzamiento en el balón medicinal (Earn y Kreaemer, 2010). Hasta la fecha, este es el primer estudio que ha evaluado parámetros de la condición física en jugadores jóvenes de pádel. Los resultados obtenidos pueden servir de referencia en la planificacióny diseño de entrenamientos de pádel(Sánchez-Alcaraz, 2014c).

\section{Referencias}

Amusa, L.O., Goon, D.T., y Amey, A.K. (2010). Gender differences in neuromotor fitness of rural South African children. Medicine in Sports, 63, 221-237.

Buchheit M., Mendez-Villanueva, A., Delhomel, G., Brughelli, M., y Ahmaidi, S. (2010) Improving repeated sprint ability in young elite soccer players: Repeated shuttle sprints vs. explosive strength training. Journal of Strength and Conditioning.Research, 24, 27152722

Castillo-Lozano, R. (2017). Epidemiology and prevention strategies for the musculoskeletal injuries in the paddle-tennis senior players. Science \& Sports, 32(3), e101-e106.

Courel-Ibáñez, J., y Sánchez-Alcaraz, B.J. (2017). Efecto de las variables situacionales sobre los puntos en jugadores de pádel de élite. Apunts, Educación Física y Deportes, 127, 68-74.

Courel-Ibáñez, J., Sánchez-Alcaraz, B.J., Cañas, J. (2015). Effectiveness at the net as a predictor of the final match outcome in professional padel players. International Journal of Performance Analysis in Sport, 15, 632-640.

Courel-Ibáñez, J., Sánchez-Alcaraz, B.J., y Cañas, J. (2016). Valoración de la precisión de golpeo en jugadores de pádel en función de su nivel de juego. Revista Internacional de Ciencias del Deporte, 45(XII), 324-333.

Courel-Ibáñez, J., Sánchez-Alcaraz, B.J., y Cañas, J. (2017). Game performance and length of rally in professional padel players. Journal of Human Kinetics, 55, 161-169.

Courel-Ibáñez, J., Sánchez-Alcaraz, B. J., García-Benítez, S., y Echegaray, M. (2017). Evolución del pádel en España en función del género y edad de los practicantes. Cultura, Ciencia y Deporte, 34(12), 39-46.

Courel-Ibáñez, J., Sánchez-Alcaraz, B. J., y Muñoz, D. (2017). Exploring game dynamics in padel. Implications for assessment and training. The Journal of Strength \& Conditioning Research. doi: 10.1519/JSC.0000000000002126.

Earp, J. E., y Kraemer, W. J. (2010). Medicine ball training implications for rotational power sports. Strength \& Conditioning Journal, 32(4), 20-25.

Federación Internacional de Pádel. (2016). Lista de paises asociados a la Federación Internacional de Pádel (FIP). Recuperado el 4 de Febrero de 2017, de http://www.padelfip.com/federaciones.

Fernandez-Fernandez, J., Ulbricht, A., y Ferrauti, A. (2014). Fitness testing of tennis players: How valuable is it?. British Journal of Sports Medicine, 48(Suppl 1), i22-i31.

Franco-Márquez F., Rodríguez-Rosell, D., González-Suárez, J.M., Pareja-Blanco, F., Mora-Custodio, R., Yañez-García, J.M., y González-Badillo, J.J. (2015) Effects of Combined Resistance Training and Plyometrics on Physical Performance in Young Soccer Players. International Journal of Sports Medicine, 36, 906-914.

Gabín, B., Camerino, O., Anguera, M.T., y Castañer, M. (2012). Lince: multiplatform sport analysis software.Procedia Computer 
Science Technology, 46, 4692-4694.

García-Benítez, S., Courel-Ibáñez, J., Pérez-Bilbao, T., y Felipe, J.L. (in press). Game responses during young padel match play: age and sex comparisons. The Journal of Strength \& Conditioning Research.

García-Ferrando, M., y Llopis, R. (2010). Ideal democrático y bienestar personal. Los hábitos deportivos en España 2010. Madrid: Consejo Superior de Deportes y Centro de Investigaciones Sociológicas.

Glazier, P.S. (2010). Game, set and match? Substantive issues and future directions in performance analysis. Sports Medicine, 40, 625-634.

Hernández, J. (2003). Relación entre diferentes pruebas de campo: fuerza, potencia y velocidad. Revista de Ciencias del Ejercicio y la Salud, 3(1), 1-10.

Junior, C.J.S., Palma, A., Imbiriba, L.A., Assis, M.R., y Barbosa, M.A.M. (2015). Relationship between relative age effect and physical characteristics of young soccer players. Cultura, Ciencia y Deporte, 10, 227-233.

Maquirriain, J., Baglione, R., y Cardey, M. (2016). Male professional tennis players maintain constant serve speed and accuracy over long matches on grass courts. European Journal of Sports Sciences, $16,845-849$.

Ministerio de Educación Cultura y Deportes (2015). Encuesta de hábitos deportivos en España 2015. Madrid: MECD.

Muñoz, D., Courel-Ibáñez, J., Sánchez-Alcaraz, B.J., Díaz, J., Grijota, F.J., y Muñoz, J. (2017). Análisis del uso y eficacia del globo para recuperar la red en función del contexto de juego en pádel. Nuevas Tendencias en Educación Física y Deportes, 31, 19-22.

Muñoz,D., Sánchez-Alcaraz, B.J., Courel-Ibáñez, J., Romero, E., Grijota, F.J., y Díaz, J. (2016). Estudio sobre el perfil y distribución de las pistas de pádel en la Comunidad Autónoma de Extremadura. EBalonmano.com: Revista de Ciencias del Deporte, 12(3), 223-230.

Priego, J. I., Olaso, J., Llanna, S., Pérez, P., González, J. C., y Sanchís, M. (2013). Padel:A Quantitative study of the shots and movements in the high-performance. Journal of Human Sport and Exercise 8(4) 925-931.

Sánchez-Alcaraz, B.J. (2013). Historia del pádel. Materiales para la historia del deporte, 11, 57-60.

Sánchez-Alcaraz, B.J.(2014a). Diferencias en las acciones de juego y la estructura temporal entre el pádel masculino y femenino profesional. Acción Motriz, 12, 17-22.

Sánchez-Alcaraz, B.J. (2014b). Análisis de la exigencia competitiva del pádel en jóvenes jugadores. Kronos, 13(1), 1-7.

Sánchez-Alcaraz, B.J.(2014c). Recomendaciones para el entrenamiento de la condición física en jóvenes jugadores de pádel. TRANCES. Revista de transmisión del conocimiento educativo y de la salud, 6 (4), 189-200.
Sánchez-Alcaraz, B.J., Cañas, J., y Courel-Ibáñez, J. (2015). Análisis de la investigación científica en pádel. AGON. International Journal of Sport Sciences, 5 (1), 44-54.

Sánchez-Alcaraz, B.J., Courel-Ibáñez, J., y Cañas, J. (2018). Estructura temporal, movimientos en pista y acciones de juego en pádel: Revisión sistemática. Retos, Nuevas Tendencias en Educación Física y Deportes, 33, 129-133.

Sánchez-Alcaraz, B.J., y Gómez-Mármol, A. (2015). Revisión de los parámetros de juego en pádel. TRANCES. Revista de transmisión del conocimiento educativo y de la salud, 7(3), 407-416.

Sánchez-Alcaraz, B.J., López-Sánchez, GF., y Pagán, A. (2016). Diferencias en la agilidad de jóvenes tenistas en función de su nivel de juego. ITF, Coaching and Sport Science Review, 69(24), 19-20.

Sánchez-Pay, A., Torres, G., y Palao, J.M. (2011). Revisión y análisis de los test físicos empleados en tenis. Motricidad. European Journal of Human Movement, 26, 105-122.

Sánchez-Alcaraz, B.J., Sánchez-Pay, A., Gómez-Mármol, A., MolinaSaorín, J., y Bazaco, M.J. (2017). Differences in forms of organization of the padel lessons with students. Revista Internacional de Medicina y Ciencias de la Actividad Física y el Deporte, 17 (67), 467-479.

Raudsepp, L. Paasuke, M. (1995). Gender differences in fundamental movement patterns, motor performances, and strength measurements of prepubertal children. Pediatric Exercise Science, 7, 294-304

Reid, M., Quinn, A., y Crespo, M. (2003). Strength and conditioning for tennis. International Tennis Federation.

Rivilla-García, J., Navarro, F., Grande, I., y Sampedro, J. (2012). Capacidad de lanzamiento en balonmano en función del puesto específico. Revista Internacional de Medicina y Ciencias de la Actividad Física y el Deporte, 12 (48), 699-714.

Roetert, P., y Ellenbecker, T.S. (2007). Complete conditioning for tennis. Human Kinetics Publishers.

Torres-Luque, G., Ramírez,A., Cabello-Manrique, D., Nikolaidis, P.T., y Alvero-Cruz, J.R. (2015). Match analysis of elite players during paddle tennis competition. International Journal of Performance Analysis in Sport, 15, 1135-1144.

Tomczak, M., y Tomczak, E. (2014). The need to report effect size estimates revisited. An overview of some recommended measures of effect size. Trends in Sport Sciences, 21(1), 19-25.

Yanci, J. y Los Arcos, A. (2015) ¿Cómo influyen la edad y el género en la capacidad de cambiar de dirección en alumnos de educación primaria? Retos, Nuevas Tendencias en Educación Física y Deportes, 28, 40-43.

Yanci, J. Los Arcos, A. Grande, I. Gil, E. Cámara, J. (2014). Correlation between agility and sprint according to student age. Collegium Antropologicum, 38(2), 533-538.
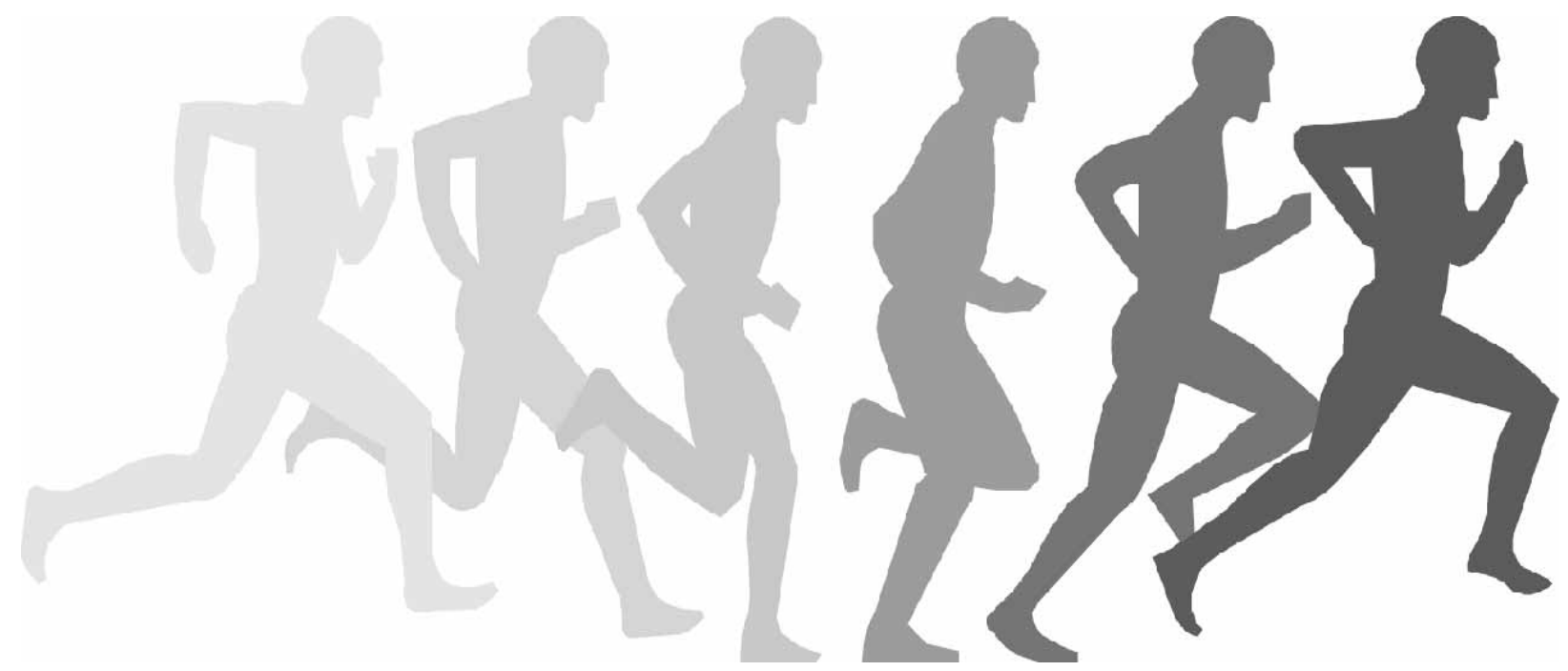